\title{
Volume-dependent electron localization in ceria
}

\author{
Sergiu Arapan, Sergey Simak and Natalia V. Skorodumova
}

\section{Linköping University Post Print}

\section{Tweet}

N.B.: When citing this work, cite the original article.

Original Publication:

Sergiu Arapan, Sergey Simak and Natalia V. Skorodumova, Volume-dependent electron localization in ceria, 2015, Physical Review B. Condensed Matter and Materials Physics, (91), $12,125108$.

http://dx.doi.org/10.1103/PhysRevB.91.125108

Copyright: American Physical Society

http://www.aps.org/

Postprint available at: Linköping University Electronic Press

http://urn.kb.se/resolve?urn=urn:nbn:se:liu:diva-116953 


\title{
Volume-dependent electron localization in ceria
}

\author{
Sergiu Arapan, ${ }^{1,2,{ }^{*}}$ Sergei I. Simak, ${ }^{3}$ and Natalia V. Skorodumova ${ }^{4,5}$ \\ ${ }^{1}$ Computational Materials Science Unit, National Institute for Materials Science, 1-1 Namiki, Tsukuba, Ibaraki 305-0044, Japan \\ ${ }^{2}$ Institute of Electronic Engineering and Industrial Technologies, Academy of Sciences of Moldova, Academiei 3/3, \\ MD-2028 Chişinău, Moldova \\ ${ }^{3}$ Department of Physics, Chemistry and Biology (IFM), Linköping University, SE-581 83 Linköping, Sweden \\ ${ }^{4}$ Multiscale Materials Modeling, Materials Science and Engineering, School of Industrial Engineering and Management, \\ KTH - Royal Institute of Technology, Brinellvägen 23, 10044 Stockholm, Sweden \\ ${ }^{5}$ Department of Physics and Astronomy, Division of Materials Theory, Uppsala University, Box 516, 75120 Uppsala, Sweden
}

(Received 14 January 2015; published 3 March 2015)

\begin{abstract}
We have performed a numerical study of the process of electron localization in reduced ceria. Our results show that different localized charge distributions can be attained in a bulk system by varying the lattice parameter. We demonstrate that the effect of electron localization is mainly determined by lattice relaxation and an accurate account for the effects of electronic correlation is necessary to achieve localized charge distribution.
\end{abstract}

DOI: 10.1103/PhysRevB.91.125108

PACS number(s): 71.15.Mb, 71.27.+a, 71.38.Ht

\section{INTRODUCTION}

Ceria is known to be an essential constituent of catalytic converters due to its excellent redox properties. The oxidation performance of ceria is determined by its ability to store, release, and transport oxygen ions. Under reduction conditions, ceria easily releases oxygen, forming a series of reduced cerium oxides. In an oxygen-rich ambience, these reduced oxides transform back to ceria by healing the oxygen vacancies during oxidation. Thus, ceria undergoes a reversible transition and is able to balance the supply of oxygen ions during a catalytic reaction. In a stoichiometric cerium oxide, each cerium ion transfers its four valence electrons, $6 s^{2} 5 d^{1} 4 f^{1}$, into the $p$ band of oxygen. When an oxygen atom is removed, the two electrons, used to occupy its $p$ orbitals, are left in the crystal. In perfect ceria, they choose to localize at the $4 f$ state of cerium atoms; thus, the formation of an oxygen vacancy is accompanied by the change of the oxidation state of two cerium atoms from +4 to $+3[1,2]$.

Chemical reactions during the redox processes occur at the surface of the metal oxides and the reactivity is determined by surface defects. In the case of ceria, such defects are oxygen vacancies. Quite extensive research, both experimental and theoretical, has been carried out to elucidate the nature of oxygen vacancies at the surface of cerium oxide. As the formation of oxygen vacancies occurs at the same time as electron localization at $\mathrm{Ce}$ atoms, oxygen vacancy formation in ceria can be monitored by measuring the $\mathrm{Ce}^{3+} / \mathrm{Ce}^{4+}$ ratio [3]. The analysis of scanning tunneling microscopy (STM) images complemented by density functional theory (DFT) calculations $[3,4]$ have suggested that electrons should localize at the $\mathrm{Ce}$ atoms neighboring the oxygen vacancy and that defects that expose $\mathrm{Ce}^{3+}$ ions to gas-phase reactants will form. This finding confirms an earlier result, which suggested that the most stable configuration of the two $\mathrm{Ce}^{3+}$ ions $\left(\mathrm{Ce}^{3+}-\mathrm{Ce}^{3+}\right)$ is next to the oxygen vacancy [1]. Experimental studies have also shown evidence of an ordering of oxygen vacancies on reduced ceria surface [5]. The defect formation

\footnotetext{
*sergiu.arapan@gmail.com
}

pattern could be satisfactorily explained by assuming charge localization at cerium ions, which are nearest neighbors to the oxygen vacancy. Recently, however, DFT calculations, which explicitly considered $\mathrm{Ce}^{3+}-\mathrm{Ce}^{3+}$ configurations on the ceria (111) surface with reduced $\mathrm{Ce}$ ions in different coordination shells, have shown that electron localization at more distant cerium ions is energetically more favorable as compared to the nearest-neighbor configuration [6,7]. A combined STM and DFT study of defective ceria film revealed the presence of $\mathrm{Ce}^{3+}$ ions in different coordination shells of the oxygen vacancy [8]. The variety of reported data poses a natural question of whether the electron localization pattern can change depending on the presence of surface defects, quality of reduced ceria films, and details of the surface preparation process. Since the charge localization is strongly correlated with the relaxation of the lattice, it may also depend on whether or not the ceria film is under stress. It is very natural to suggest that it might also depend on the system volume, one of the main thermodynamic parameters.

Theoretical tools allow us to separate different factors, which might determine or influence the localized charge distribution. Therefore, in this work, we study the bulk volume dependence of electron localization in reduced ceria using DFT-based computation methods. It turns out that, depending on pressure, different localized charge distributions can be attained.

\section{CAlCulation Details}

\section{A. Parameters of DFT calculations}

There exist several theoretical works related to the problem of accurate description of the electronic structure and the physical properties of stoichiometric $\mathrm{CeO}_{2}$, reduced $\mathrm{CeO}_{2-x}$, and $\mathrm{Ce}_{2} \mathrm{O}_{3}$ oxides [1,4,9-11]. Standard DFT using local density approximation (LDA) or generalized gradient approximation (GGA) for the exchange-correlation part of the energy functional fails to give a satisfactory description of the electronic structure of reduced ceria oxides. The reason is the presence of Coulomb self-interaction terms in these density functionals, which tend to stabilize the delocalized 
solution. To cure this shortcoming of standard DFT, one can, for example, correct the intraband Coulomb interactions by means of the Hubbard $U$ parameter [12]. Despite the fact that the Hubbard repulsion energy $U$ could be calculated from the first principles [4], in practice it plays the role of an adjustable parameter, and its value is chosen to fit the experimental observations. Thus, DFT $+U$ is a pragmatic approach, often providing an adequate description of systems with strongly correlated electrons. Another possibility to correct the improper electron self-interaction is to modify standard DFT functionals by adding some amount of nonlocal Fock exchange [13]. The use of such hybrid functionals is computationally more demanding than DFT $+U$ and, in the case of reduced ceria, they do not provide a more accurate description than the DFT $+U$ approximation [11].

For our purpose, the description of reduced cerium oxides within the DFT $+U$ formalism is sufficient [14]. Therefore, we performed our calculations using both $\mathrm{LDA}+U$ and $\mathrm{GGA}+U$ energy functionals. For the magnitude of the Hubbard parameter, we chose $U=6 \mathrm{eV}$ and $U=5 \mathrm{eV}$ for the LDA and Perdew-Burke-Ernzerhof (PBE) [15] approximations, respectively [9]. All calculations were performed using the Vienna $A b$ initio Simulation Package (VASP) [16] with projector-augmented-wave (PAW) potentials $[17,18]$. In these calculations, the $\mathrm{Ce}(4 f, 5 s, 5 p, 5 d, 6 s)$ and $\mathrm{O}(2 s, 2 p)$ electrons were treated as valence states, with the remaining electrons kept frozen in the core. In order to perform accurate ion relaxation calculations, we used a $600 \mathrm{eV}$ plane-wave energy cutoff and the internal parameters were optimized until the Hellmann-Feynman forces became less than $0.005 \mathrm{eV} / \AA$. We modeled $\mathrm{CeO}_{2-x}$ using a $2 \times 2 \times 2$ cubic cell with one oxygen vacancy, which comprised 95 ions in total. The reciprocal space was sampled by a $3 \times 3 \times 3$ Monkhorst-Pack $k$-point mesh that yielded 14 irreducible $k$ points. In all calculations, we assumed an antiferromagnetic (AFM) alignment of the spin of localized electrons, which is the ground-state magnetic configuration at $0 \mathrm{~K}$. Next to the $\mathrm{DFT}+U$ calculations, we also performed DFT calculations treating $\mathrm{Ce}^{3+}$ ions in the core state model (CSM) in the spirit of Ref. [2]. In this approach, the $4 f$ states of cerium are treated as core states. In particular,we used a GGA PAW potential especially generated to describe cerium atoms with $(5 s, 5 p, 5 d, 6 s)$ valence electrons only.

\section{B. Modeling reduced $\mathrm{CeO}_{2-x}$}

We have modeled reduced bulk ceria by removing an $\mathrm{O}$ ion from the $2 \times 2 \times 2$ cubic supercell, as shown in Fig. 1 . This corresponds to the vacancy concentration of about $1.5625 \%$. The $\mathrm{O}$ vacancy is surrounded by four nearest-neighbor (NN) $\mathrm{Ce}$ ions, labeled by numerals, and 12 next-nearest-neighbor (NNN) Ce ions, labeled by numerals with prime. We have considered the following three types of $\mathrm{Ce}^{3+}-\mathrm{Ce}^{3+}$ configurations:

(1) $\mathrm{Ce}_{n}^{3+} \mathrm{Ce}_{m}^{3+}$ : both reduced $\mathrm{Ce}$ ions are in the first coordination sphere $(\mathrm{NN})$ of the $\mathrm{O}$ vacancy.

(2) $\mathrm{Ce}_{n}^{3+} \mathrm{Ce}_{m^{\prime}}^{3+}$ : one reduced $\mathrm{Ce}$ ion is within the first coordination sphere (NN) and another one is in the second coordination shell (NNN) of the $\mathrm{O}$ vacancy.

(3) $\mathrm{Ce}_{n^{\prime}}^{3+} \mathrm{Ce}_{m^{\prime}}^{3+}$ : both reduced $\mathrm{Ce}$ ions are in the second coordination shell (NNN) of the $\mathrm{O}$ vacancy.

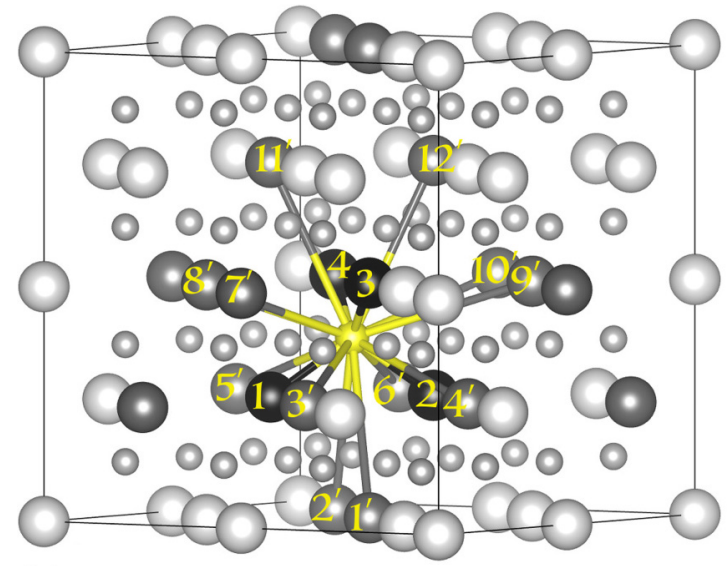

(a)
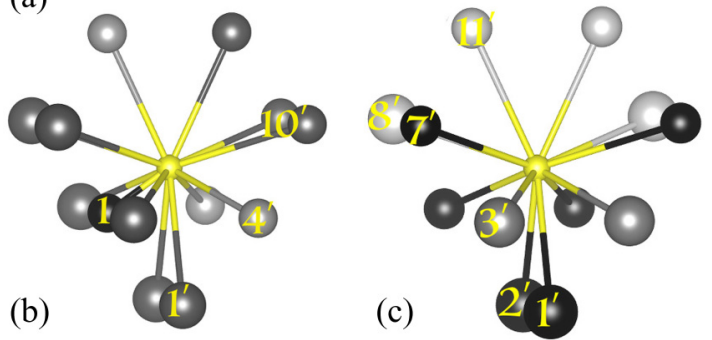

FIG. 1. (Color online) (a) A cubic supercell (small balls: O; large balls: Ce) used to model reduced ceria with $(2 \times 2 \times 2)$ oxygen vacancy periodicity (periodical images are also shown). For a given oxygen vacancy (yellow ball), nearest-neighbor Ce ions (dark gray) and next-nearest-neighbor Ce ions (gray) are labeled. (b) $\mathrm{Ce}^{3+}$ ions (differentiated by size and grayscale level) which belong to different groups of $\mathrm{Ce}_{1}^{3+} \mathrm{Ce}_{m^{\prime}}^{3+}$ configurations. (c) $\mathrm{Ce}^{3+}$ ions (differentiated by size and grayscale level) which belong to different groups of $\mathrm{Ce}_{1^{\prime}}^{3+} \mathrm{Ce}_{m^{\prime}}^{3+}$ configurations.

Due to the symmetry of the cubic supercell, the number of different reduced ceria structures is much less than all possible $\mathrm{Ce}_{n}^{3+} \mathrm{Ce}_{m}^{3+}$ pairs, where $n, m$ go through the set of the $\mathrm{NN}$ and NNN Ce ions. In particular, we can consider only one pair, $\mathrm{Ce}_{1}^{3+} \mathrm{Ce}_{2}^{3+}$, for reduced $\mathrm{CeO}_{2-x}$ with both reduced $\mathrm{Ce}^{3+}$ ions $\mathrm{NN}$ of the $\mathrm{O}$ vacancy. The symmetry analysis shows that there are only three different groups for $\mathrm{Ce}_{n}^{3+} \mathrm{Ce}_{m^{\prime}}^{3+}, n=1, m^{\prime}=$ $1^{\prime}, 4^{\prime}, 10^{\prime}$ and five different groups for $\mathrm{Ce}_{n^{\prime}}^{3+} \mathrm{Ce}_{m^{\prime}}^{3+}, n^{\prime}=1^{\prime}, m^{\prime}=$ $2^{\prime}, 3^{\prime}, 7^{\prime}, 8^{\prime}, 10^{\prime}$ configurations.

The localization of an electron at a given cerium atom, in the cases considered here, is similar to the formation of a small polaron. The electron-lattice interaction induces a lattice distortion that forms a trapping potential for the electron. Therefore, an accurate description of local deformations is essential in order to obtain the electron localization in reduced ceria. To get an appropriate initial pattern of local distortions, we performed calculations using the CSM approach. The optimization of internal parameters in this scheme distorts the lattice in a fashion that ensures the charge localization at a chosen site. This approach is physically grounded and is quite robust. In all of our calculations, we have gotten electrons localized at given sites when starting our DFT $+U$ calculations from the structures obtained in the CSM calculations.

Another difficulty of an accurate description of localization is to obtain a stable self-consistent solution when applying the 
$U$ parameter to the $f$ shell. Here we follow the $U$-ramping scheme [19] by obtaining a converged self-consistent solution at $U=0$ and propagating it up to a finite value of $U$.

\section{RESULTS AND DISCUSSION}

\section{A. Equation of state and enthalpy}

We performed a set of $\mathrm{PBE}+U$ calculations at different volumes in order to get the equation of state (EOS) $E=E(V)$ for various $\mathrm{Ce}_{n}^{3+} \mathrm{Ce}_{m}^{3+}$ configurations. For each $\mathrm{Ce}^{3+}-\mathrm{Ce}^{3+}$ configuration, we first obtained a stable solution at a given volume $V$. Then, we performed structure optimization at finite $U$ and the obtained atom positions as well as the charge density optimized for volume $V$ were further used as a starting configuration and charge distribution for volume $V+\Delta V$. In order to check that at different volumes the electronic solution converged to the same ground state, the $U$-ramping procedure for a set of volumes with a subsequent structure optimization was performed for several $\mathrm{Ce}^{3+}-\mathrm{Ce}^{3+}$ configurations. These tests showed that the obtained solutions were stable. In Fig. 2, we present the results of our PBE $+U$ calculations. In Fig. 2(a), the volume dependence of the energies of various $\mathrm{Ce}_{n}^{3+} \mathrm{Ce}_{m}^{3+}$ structures relative to the energy of the $\mathrm{Ce}_{1^{\prime}}^{3+} \mathrm{Ce}_{2^{\prime}}^{3+}$ configuration, which is stable at ambient pressure, is shown.

We observe a quite remarkable dependence of energy on volume for different types of reduced ceria configurations. In particular, at large volumes, corresponding to $10 \%$ volume extension, we have the following ordering of the $\mathrm{Ce}^{3+}-\mathrm{Ce}^{3+}$ configurations: lowest in energy are structures with the two $\mathrm{Ce}^{3+}$ ions in the second coordination shell of the $\mathrm{O}$ vacancy, followed by structures with $\mathrm{Ce}^{3+}$ ions in both first and second coordination shells, and the least stable is the configuration where both $\mathrm{Ce}^{3+}$ ions are in the first coordination sphere of the $\mathrm{O}$ vacancy. If we look at small volumes, for example, that correspond to $10 \%$ volume compression, we would see a reversed picture: the lowest in energy is the $\mathrm{Ce}_{1}^{3+} \mathrm{Ce}_{2}^{3+}$ structure, followed by $\mathrm{Ce}_{1}^{3+} \mathrm{Ce}_{m^{\prime}}^{3+}$, and then by $\mathrm{Ce}_{1^{\prime}}^{3+} \mathrm{Ce}_{m^{\prime}}^{3+}$ configurations. At ambient pressure, which corresponds to the lattice parameter of $5.507 \AA$, the
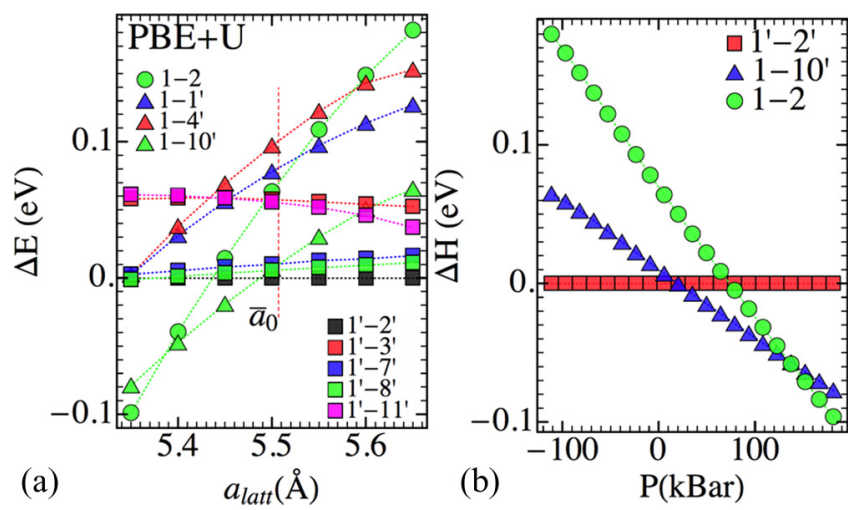

FIG. 2. (Color online) (a) Energy vs volume for all distinct reduced ceria structures relative to the energy of the $\mathrm{Ce}_{1^{\prime}}^{3+} \mathrm{Ce}_{2^{\prime}}^{3+}$ configuration. (b) Dependence of enthalpy on pressure for $\mathrm{Ce}_{1}^{3+} \mathrm{Ce}_{2}^{3+}$, $\mathrm{Ce}_{1}^{3+} \mathrm{Ce}_{10^{\prime}}^{3+}$ structures with respect to the enthalpy of the $\mathrm{Ce}_{1^{\prime}}^{3+} \mathrm{Ce}_{2^{\prime}}^{3+}$ configuration.
TABLE I. Parameters of the Birch-Murnaghan equation of state averaged over different $\mathrm{Ce}_{n}^{3+} \mathrm{Ce}_{m}^{3+}$ configurations of reduced ceria for different types of calculations. Data shown in parentheses are the corresponding standard deviations.

\begin{tabular}{lccc}
\hline \hline & $\mathrm{PBE}+U$ & $\mathrm{LDA}+U$ & $\mathrm{CSM}(\mathrm{GGA})$ \\
\hline$a_{0}(\AA)$ & $5.5069(0.0006)$ & $5.4173(0.0006)$ & $5.4854(0.0004)$ \\
$V_{0}\left(\AA^{3}\right)$ & $1336.028(0.440)$ & $1271.865(0.412)$ & $1320.446(0.279)$ \\
$B_{0}(\mathrm{GPa})$ & $174.746(0.123)$ & $204.762(0.078)$ & $167.385(0.104)$ \\
$B^{\prime}$ & $4.518(0.019)$ & $4.440(0.005)$ & $4.356(0.004)$ \\
\hline \hline
\end{tabular}

most stable configuration is $\mathrm{Ce}_{1^{\prime}}^{3+} \mathrm{Ce}_{2^{\prime}}^{3+}$. The parameters of the third-order Birch-Murnaghan equation of state, averaged over different $\mathrm{Ce}^{3+}-\mathrm{Ce}^{3+}$ structures, are given in Table I. The average equilibrium lattice parameter of the reduced ceria is slightly larger than the equilibrium lattice parameter of perfect ceria (about $5.495 \AA$;) calculated within the PBE $+U$ method with $U=5 \mathrm{eV}$ [9], which is consistent with experimental observations [20]. However, at a moderate compression (20 kbar) [Fig. 2(b)], the $\mathrm{Ce}_{1}^{3+} \mathrm{Ce}_{10^{\prime}}^{3+}$ configuration becomes stable. This corresponds to a lattice parameter of $5.486 \AA$, which differs from the equilibrium parameter by less than $0.4 \%$. At a pressure of $74 \mathrm{kbar}$, the structure with both reduced $\mathrm{Ce}$ ions in the first sphere of the $\mathrm{O}$ vacancy becomes energetically more favorable than structures with both $\mathrm{Ce}^{3+}$ ions in the second coordination shell. This transition occurs for a lattice parameter of $5.435 \AA$, which differs from the equilibrium lattice parameter by $1.3 \%$. Finally, the $\mathrm{Ce}_{1}^{3+} \mathrm{Ce}_{2}^{3+}$ structure becomes stable at a pressure of $135 \mathrm{kbar}$, which corresponds to the lattice parameter of $5.385 \AA$, which is $2.2 \%$ smaller than the lattice parameter at ambient pressure.

In order to understand how the stability of different configurations depends on the energy functional, we performed similar calculations using $\mathrm{LDA}+U$. The results for the energy and enthalpy differences are shown in Fig. 3. The parameters of the third-order Birch-Murnaghan EOS are given in Table I. The equilibrium lattice parameter, averaged over all reduced ceria configurations, is $5.417 \AA$, which is expectedly lower than that obtained in the PBE $+U$ calculations, as LDA usually
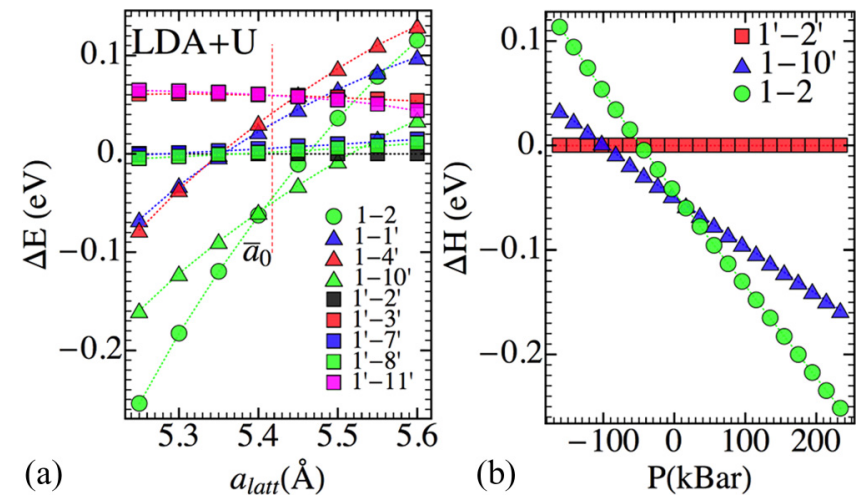

FIG. 3. (Color online) (a) Dependence of energy on volume for all distinct reduced ceria structures relative to the energy of the $\mathrm{Ce}_{1^{\prime}}^{3+} \mathrm{Ce}_{2^{\prime}}^{3+}$ configuration for the LDA $+U$ type of calculations. (b) Enthalpy vs pressure for $\mathrm{Ce}_{1}^{3+} \mathrm{Ce}_{2}^{3+}, \mathrm{Ce}_{1}^{3+} \mathrm{Ce}_{10^{\prime}}^{3+}$ structures with respect to the enthalpy of the $\mathrm{Ce}_{1^{\prime}}^{3+} \mathrm{Ce}_{2^{\prime}}^{3+}$ configuration. 
underestimates the equilibrium lattice parameter. Overall, the picture in Fig. 3(a) looks similar to the $\mathrm{PBE}+U$ case [Fig. 2(a)], with the energy curves shifted with respect to the equilibrium lattice parameter $a_{0}$. At increased volumes, the $\mathrm{Ce}_{n^{\prime}}^{3+} \mathrm{Ce}_{m^{\prime}}^{3+}$ structure tends to be more stable than the other configurations. At high pressure, the order is reversed, with the $\mathrm{Ce}_{n}^{3+} \mathrm{Ce}_{m}^{3+}$ configuration being most stable, followed by $\mathrm{Ce}_{n}^{3+} \mathrm{Ce}_{m^{\prime}}^{3+}$ and $\mathrm{Ce}_{n^{\prime}}^{3+} \mathrm{Ce}_{m^{\prime}}^{3+}$ structures. At the equilibrium volume, the $\mathrm{Ce}_{1}^{3+} \mathrm{Ce}_{10^{\prime}}^{3+}$ configuration, with $\mathrm{Ce}^{3+}$ ions in the first and second coordination shells, is stable but the $\mathrm{Ce}_{1}^{3+} \mathrm{Ce}_{2}^{3+}$ structure follows with a narrow difference. Indeed, at a small pressure of $10 \mathrm{kbar}$, the configuration of reduced ceria with the two $\mathrm{Ce}^{3+}$ ions in the vicinity of the $\mathrm{O}$ vacancy becomes energetically more favorable. This transition occurs for a negligible change in the lattice parameter $(0.2 \%)$, at $5.408 \AA$. With the volume expansion, the $\mathrm{Ce}_{1^{\prime}}^{3+} \mathrm{Ce}_{2^{\prime}}^{3+}$ structure becomes more stable than the configuration with both $\mathrm{Ce}^{3+}$ in the first coordination sphere at $5.460 \AA$ ( $0.8 \%$ change, $P=-48 \mathrm{kbar})$, and the configuration with $\mathrm{Ce}^{3+}$ in the first and second coordination shells at $5.515 \AA$ ( $1.8 \%$ change of the lattice parameter, $P=-98 \mathrm{kbar})$. One can notice that the enthalpy curves in Figs. 2(b) and 3(b) are shifted with respect to each other by $120 \mathrm{kbar}$.

As stated above, the CSM calculations are able to provide appropriate lattice distortions. Therefore, it is interesting to see how the energies of the different $\mathrm{Ce}^{3+}-\mathrm{Ce}^{3+}$ configurations calculated within CSM vary with changing volume and how they compare to the results of $\mathrm{DFT}+U$ calculations. The results of such calculations are summarized in Fig. 4. We notice that the trends obtained in the CSM calculations are similar to those from $\mathrm{DFT}+U$, i.e., larger pressure is required to induce the transitions between different reduced ceria configurations. We exploited this similarity to analyze the effect of relaxation for different structures.

\section{B. Energy contributions to the total energy}

To understand the driving force behind the observed transitions between different $\mathrm{Ce}^{3+}-\mathrm{Ce}^{3+}$ configurations, it can be instructive to analyze different contributions to the total energy and their volume dependences.
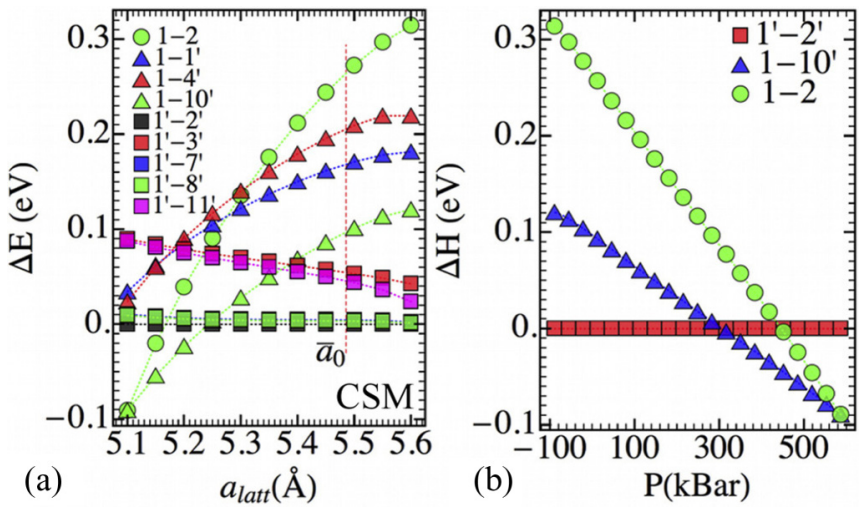

FIG. 4. (Color online) (a) Dependence of energy vs volume for all distinct reduced ceria structures relative to the energy of the $\mathrm{Ce}_{1^{\prime}}^{3+} \mathrm{Ce}_{2^{\prime}}^{3+}$ configuration for the CSM calculations. (b) Dependence of enthalpy vs pressure for $\mathrm{Ce}_{1}^{3+} \mathrm{Ce}_{2}^{3+}, \mathrm{Ce}_{1}^{3+} \mathrm{Ce}_{10^{\prime}}^{3+}$ structures with respect to the enthalpy of the $\mathrm{Ce}_{1^{\prime}}^{3+} \mathrm{Ce}_{2^{\prime}}^{3+}$ configuration.

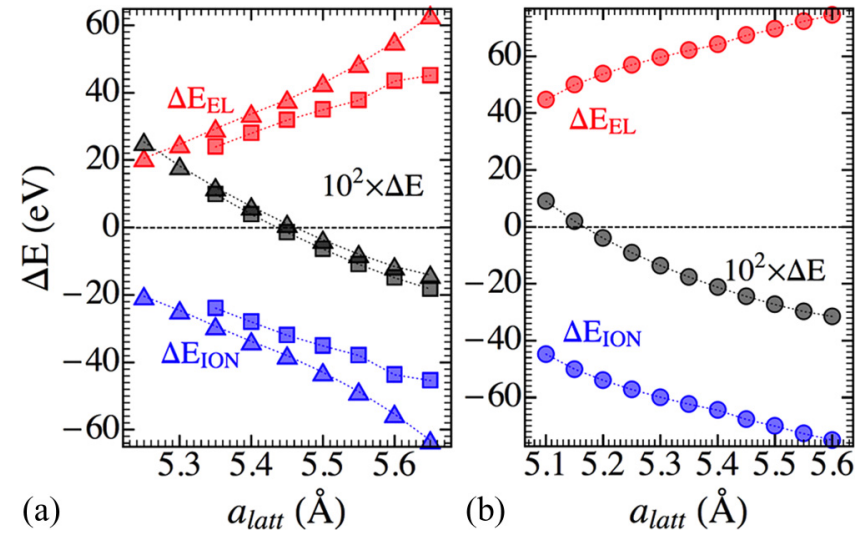

FIG. 5. (Color online) Contributions of the ionic energy term (blue), $\Delta E_{\mathrm{ion}}$, and of the electronic subsystem (red), $\Delta E_{\mathrm{el}}$, to the total energy difference (black), $\Delta E$, between two reduced

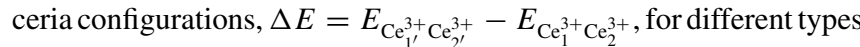
of calculations: (a) $\mathrm{PBE}+U(\mathbf{\square})$ and $\mathrm{LDA}+U$ ( $\mathbf{\Delta})$, (b) GGA $\operatorname{CSM}(\bullet)$.

Our analysis suggests that various energy terms could be combined in two groups that have similar volume dependence for all types of calculations: one which describes the electrostatic interaction between ions $\left(E_{\mathrm{ion}}\right)$, and the second one comprising the rest of the energy terms $\left(E_{\mathrm{el}}\right)$, which describes the electronic subsystem (which also contains the electrostatic interaction between valence electrons and lattice ions). In Fig. 5, we show how these different contributions to the total energy difference between $\mathrm{Ce}_{1^{\prime}}^{3+} \mathrm{Ce}_{2^{\prime}}^{3+}$ and $\mathrm{Ce}_{1}^{3+} \mathrm{Ce}_{2}^{3+}$ configurations depend on the lattice parameter. All three types of calculations $(\mathrm{GGA}+U, \mathrm{LDA}+U$, and CSM) show

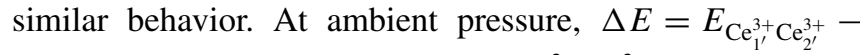
$E_{\mathrm{Ce}_{1}^{3+} \mathrm{Ce}_{2}^{3+}}<0$ and the stability of $\mathrm{Ce}_{1^{\prime}}^{3+} \mathrm{Ce}_{2^{\prime}}^{3+}$ is determined by the ionic energy contribution. One may observe that for the whole volume range, the electrostatic energy of the lattice is lower for the configuration with the two $\mathrm{Ce}^{3+}$ ions being in the second coordination shell [6]. The energy of the electronic subsystem, however, favors the structure with the $\mathrm{Ce}^{3+}$ ions in the first coordination sphere. These two contributions to the total energy difference $\Delta E=\Delta E_{\mathrm{ion}}+\Delta E_{\mathrm{el}}$ have similar behavior with pressure, namely, the energy difference between the two structures $\left(\Delta E_{\mathrm{ion}}, \Delta E_{\mathrm{el}}\right)$ becomes smaller with decreasing volume but has opposite signs. There is a competition between $\Delta E_{\text {ion }}$ and $\Delta E_{\mathrm{el}}$. The applied pressure tries to lower the ionic energy of the $\mathrm{Ce}_{1}^{3+} \mathrm{Ce}_{2}^{3+}$ structure, but at the same time lower the energy of the electronic subsystem of the $\mathrm{Ce}_{1}^{3+} \mathrm{Ce}_{10^{\prime}}^{3+}$ structure. The decrease in energy is faster for the ionic contribution, so the transition occurs when $\left|\Delta E_{\mathrm{ion}}\right|<\Delta E_{\mathrm{el}}$. A similar picture emerges when comparing the energies of the $\mathrm{Ce}_{1}^{3+} \mathrm{Ce}_{2}^{3+}$ structure with that of $\mathrm{Ce}_{1}^{3+} \mathrm{Ce}_{10^{\prime}}^{3+}$.

\section{Ionic relaxation}

As structural distortions around the vacancy play an important role in the stabilization of a particular $\mathrm{Ce}^{3+}-\mathrm{Ce}^{3+}$ configuration, we analyze the obtained local deformations in more detail. In particular, we compare the positions of ions before, $\mathbf{R}_{i}^{0}$, and after, $\mathbf{R}_{i}$, the relaxation with respect to the 


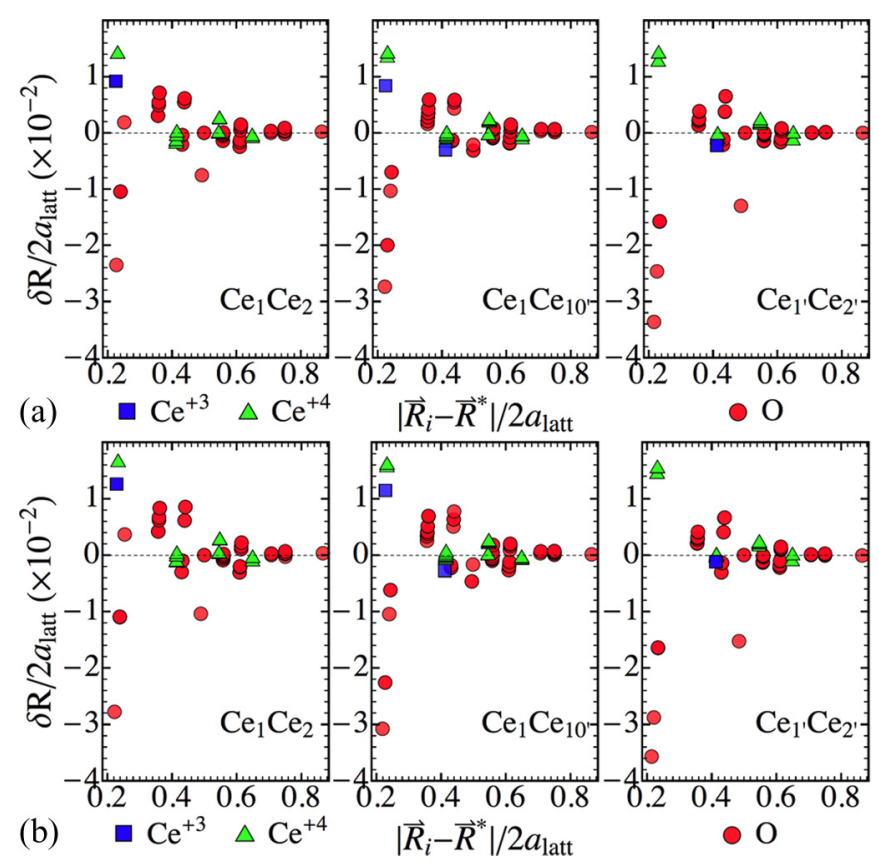

FIG. 6. (Color online) Displacements of various ions within the simulation cell with respect to the position of the $\mathrm{O}$ vacancy for relaxed $\mathrm{Ce}_{1}^{3+} \mathrm{Ce}_{2}^{3+}, \mathrm{Ce}_{1}^{3+} \mathrm{Ce}_{10^{\prime}}^{3+}$, and $\mathrm{Ce}_{1^{\prime}}^{3+} \mathrm{Ce}_{2^{\prime}}^{3+}$ configurations at (a) $a_{\mathrm{latt}}=5.35 \AA$ and (b) $a_{\mathrm{latt}}=5.50 \AA$. Displacements corresponding to $\mathrm{Ce}^{+3}, \mathrm{Ce}^{+4}$, and $\mathrm{O}^{-2}$ ions are shown by squares, triangles, and circles, respectively (some data overlap). For $\delta R<0(\delta R>0)$, ions move towards (away from) the vacancy site.

position of the O vacancy, $\mathbf{R}^{*}$. In Fig. 6, we show the shifts of ions with respect to the vacancy, $\delta R=\left|\mathbf{R}_{i}-\mathbf{R}^{*}\right|-\mid \mathbf{R}_{i}^{0}-$ $\mathbf{R}^{*} \mid$, for the PBE $+U$ type of calculations and two different lattice parameters. We notice that the $\mathrm{O}$ and $\mathrm{Ce}$ ions within the first $\mathrm{O}$ and $\mathrm{Ce}$ coordination shells of the $\mathrm{O}$ vacancy experience the largest displacements. The second $\mathrm{O}$ shell shows much smaller but still noticeable displacements. The $\mathrm{Ce}$ ions of the second coordination shell of the $\mathrm{O}$ vacancy show very little displacements upon relaxation.

The ions of the first $\mathrm{O}$ coordination shell tend to move towards the vacancy, while the Ce ions of the first Ce coordination shell move away from the vacancy. This decreases the distances between remaining oxygen atoms and cerium atoms that partially compensates for the decrease of their coordination. This trend is expectedly more pronounced when there are only $\mathrm{Ce}^{4+}$ in the first shell of the vacancy, and we see larger displacements of nearest $\mathrm{O}$ ions in this case. The change in $\mathrm{Ce}-\mathrm{O}$ bond length during structure optimization at different lattice parameters is shown in Fig. 7. As expected, O ions move away from $\mathrm{Ce}^{3+}$ towards $\mathrm{Ce}^{4+}$. Interestingly, the displacements of the $\mathrm{Ce}^{4+}$ ions within the first coordination sphere are quite inert to the change of the lattice parameter and are almost the same for different $\mathrm{Ce}^{3+}$ configurations (Fig. 6). On the contrary, the displacements of the $\mathrm{O}$ ions of the first shell are sensitive to the variation of lattice parameter. The described relaxation pattern around the vacancy is common for all methods $(\mathrm{GGA}+U, \mathrm{LDA}+U$, and $\mathrm{CSM})$ employed in our calculations.

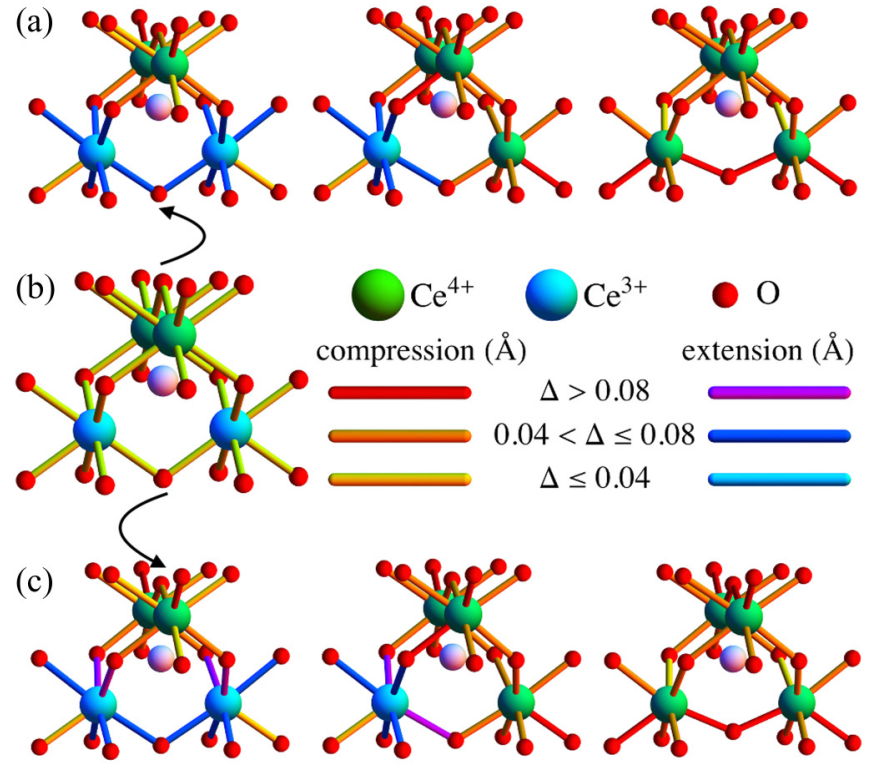

FIG. 7. (Color online) (a) Ce-O distances within the first coordination sphere of the $\mathrm{O}$ vacancy (creamy sphere) for different $\mathrm{Ce}^{3+}$ $\mathrm{Ce}^{3+}$ configurations relaxed at $a_{\text {latt }}=5.35 \AA$. (b) Ce-O distances within the first coordination of the $\mathrm{O}$ vacancy for the initial structure (same for all $\mathrm{Ce}^{3+}-\mathrm{Ce}^{3+}$ configurations, except for the positions of $\mathrm{Ce}^{3+}$ ions). $\mathrm{Ce}-\mathrm{O}$ bonds are colored according to their length: red-shifted (blue-shifted) if $\mathrm{O}$ ions move towards (away from) $\mathrm{Ce}$ ions. (c) $\mathrm{Ce}-\mathrm{O}$ distances within first coordination of the $\mathrm{O}$ vacancy for different $\mathrm{Ce}^{3+}-\mathrm{Ce}^{3+}$ configurations relaxed at $a_{\text {latt }}=5.50 \AA$.

We can try to estimate how much the relaxation of different groups of ions contributes to the total relaxation energy by performing partial lattice optimization using the CSM approach. In Fig. 8(a), the volume dependence of the relaxation energy is shown for different configurations of reduced Ce ions. In Fig. 8(b), the contributions of different groups of ions to the total relaxation energy are presented. We can see that the relaxation energy is the largest for the $\mathrm{Ce}_{1^{\prime}}^{3+} \mathrm{Ce}_{2^{\prime}}^{3+}$ configuration, with both reduced $\mathrm{Ce}$ ions in the second coordination shell of the vacancy. It is the smallest for the $\mathrm{Ce}_{1}^{3+} \mathrm{Ce}_{2}^{3+}$ configuration, with both reduced $\mathrm{Ce}$ ions in the first coordination sphere of the $\mathrm{O}$ vacancy. For all of the configurations, the total relaxation energy decreases almost linearly with pressure. The contributions to the total relaxation energy were calculated by allowing the relaxation of a certain group of ions, so, strictly speaking, it is not the exact amount that contributes to the lattice relaxation due to these atoms, but it is a fair enough estimate. In accordance with the analysis of local distortions (Fig. 6), we see that the relaxation of the $\mathrm{Ce}$ and $\mathrm{O}$ ions from the first $\mathrm{Ce}$ and $\mathrm{O}$ coordination spheres is responsible for about $60 \%$ of the total relaxation energy. This contribution is slightly larger for the $\mathrm{Ce}^{3+}-\mathrm{Ce}^{3+}$ configurations with only one or zero $\mathrm{Ce}^{3+}$ ions within the first coordination sphere of the vacancy. This contribution is pressure dependent, showing larger values for larger lattice parameters. These facts, in accordance with the Ce-O bond length analysis in Fig. 7, emphasize the important role played by $\mathrm{O}$ ions in the relaxation. Allowing also for the relaxation of the $\mathrm{O}$ ions of the second $\mathrm{O}$ coordination shell, we get about $80 \%$ of the total relaxation energy. The relaxation of the second 


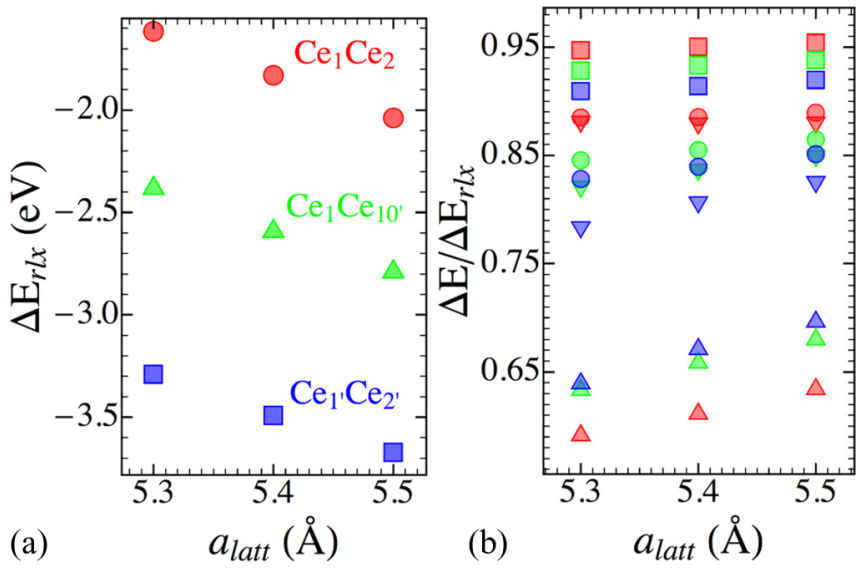

FIG. 8. (Color online) (a) Volume dependence of the energy of relaxation for three reduced ceria structures: different symbols and colors correspond to different $\mathrm{Ce}^{3+}-\mathrm{Ce}^{3+}$ configurations. (b) Contributions to the relaxation energy from different groups of ions $(\boldsymbol{\Lambda}: \mathrm{Ce}$ and $\mathrm{O}$ in the first coordination sphere of the $\mathrm{O}$ vacancy; $\boldsymbol{\nabla}$ : $\mathrm{Ce}$ in the first coordination sphere and $\mathrm{O}$ within the first two coordination shells of the vacancy; $\bullet$ : $\mathrm{Ce}$ and $\mathrm{O}$ within the first two coordination shells of the $\mathrm{O}$ vacancy; $\mathbf{\square}$ : Ce in the first two coordination shells and $\mathrm{O}$ within the first four coordination shells of the defect) in the vicinity of the $\mathrm{O}$ vacancy for different reduced ceria structures (red: $\mathrm{Ce}_{1}^{3+} \mathrm{Ce}_{2}^{3+}$; green: $\mathrm{Ce}_{1}^{3+} \mathrm{Ce}_{10^{\prime}}^{3+}$; blue: $\mathrm{Ce}_{1^{\prime}}^{3+} \mathrm{Ce}_{2^{\prime}}^{3+}$ ).

Ce coordination shell brings small changes to the relaxation energy: almost negligible for the $\mathrm{Ce}_{1}^{3+} \mathrm{Ce}_{2}^{3+}$ configuration, but finite for reduced ceria structures with $\mathrm{Ce}^{3+}$ ions in the second coordination shell of the vacancy.

\section{Conclusions}

Our study has shown that the ground-state configuration of two reduced $\mathrm{Ce}^{3+}$ ions and oxygen vacancy depends on the lattice parameter. At larger volumes, the configurations with both reduced $\mathrm{Ce}^{3+}$ ions within the second coordination shell of the $\mathrm{O}$ vacancy are favored, while at compression the structure with both $\mathrm{Ce}^{3+}$ within the first coordination sphere of the defect becomes more stable. The performed calculations show that this transition occurs for a small change of the lattice parameter around the equilibrium value. This result does not depend on the type of the energy functional used. As the LDA (GGA) functional usually underestimates (overestimates) the lattice parameter at ambient conditions, the only difference between the results obtained with $\mathrm{LDA}+U$ and $\mathrm{PBE}+U$ is the stable configuration at the equilibrium volume. It is a configuration with both $\mathrm{Ce}^{3+}$ in the second coordination shell of the $\mathrm{O}$ vacancy for the $\mathrm{PBE}+U$ approach, and a configuration with one $\mathrm{Ce}^{3+}$ in the first and one $\mathrm{Ce}^{3+}$ in the second coordination shells for LDA $+U$.

The sequence of different stable reduced ceria configurations with pressure is reproduced also by the CSM approach. It is the effect of lattice relaxation that mostly determines the electron localization at the $\mathrm{Ce} 4 f$ orbitals. Hence, we believe that an accurate account of the electronic correlation is necessary to achieve the effect of electron localization, but it is not as important in determining actual $\mathrm{Ce}^{3+}-\mathrm{Ce}^{3+}$ configurations. Our calculations have also shown that the lattice deformation due to vacancy is the largest in the first coordination of the defect. This finding agrees with previously obtained results for the (111) ceria surface [6,7] where different $\mathrm{Ce}^{3+}-\mathrm{Ce}^{3+}$ patterns were observed.

\section{ACKNOWLEDGMENTS}

Calculations were performed using PDC and NSC computer facilities. N.V.S. acknowledges the financial support from the Swedish Research Council, Swedish Energy Agency (STEM) and Carl Tryggers Foundation. S.I.S. acknowledges support from the Swedish Research Council Project No. 2014-4750 and LiLi-NFM, and the Swedish Government Strategic Research Area Grant in Materials Science to the Advanced Functional Materials (AFM) research environment at Linköping University.
[1] N. V. Skorodumova, S. I. Simak, B. I. Lundqvist, I. A. Abrikosov, and B. Johansson, Phys. Rev. Lett. 89, 166601 (2002).

[2] N. V. Skorodumova, R. Ahuja, S. I. Simak, I. A. Abrikosov, B. Johansson, and B. I. Lundqvist, Phys. Rev. B 64, 115108 (2001).

[3] F. Esch, S. Fabris, L. Zhou, T. Montini, C. Africh, P. Fornasiero, G. Comelli, and R. Rosei, Science 309, 752 (2005).

[4] S. Fabris, G. Vicario, G. Balducci, S. de Gironcoli, and S. Baroni, J. Phys. Chem. B 109, 22860 (2005).

[5] S. Torbrügge, M. Reichling, A. Ishiyama, S. Morita, and Ó. Custance, Phys. Rev. Lett. 99, 056101 (2007).

[6] M. V. Ganduglia-Pirovano, J. L. F. Da Silva, and J. Sauer, Phys. Rev. Lett. 102, 026101 (2009).

[7] H. Y. Li, H. F. Wang, X.-Q. Gong, Y.-L. Guo, Y. Guo, G. Lu, and P. Hu, Phys. Rev. B 79, 193401 (2009).

[8] J.-F. Jerratsch, X. Shao, N. Nilius, H. J. Freund, C. Popa, M. V. Ganduglia-Pirovano, A. M. Burow, and J. Sauer, Phys. Rev. Lett. 106, 246801 (2011).

[9] D. A. Andersson, S. I. Simak, B. Johansson, I. A. Abrikosov, and N. V. Skorodumova, Phys. Rev. B 75, 035109 (2007).
[10] J. L. F. Da Silva, M. V. Ganduglia-Pirovano, J. Sauer, V. Bayer, and G. Kresse, Phys. Rev. B 75, 045121 (2007).

[11] J. Kullgren, C. W. M. Castleton, C. Müller, D. M. Ramo, and K. Hermansson, J. Chem. Phys. 132, 054110 (2010).

[12] V. I. Anisimov, J. Zaanen, and O. K. Andersen, Phys. Rev. B 44, 943 (1991).

[13] A. D. Becke, J. Chem. Phys. 98, 5648 (1993).

[14] S. L. Dudarev, G. A. Botton, S. Y. Savrasov, C. J. Humphreys, and A. P. Sutton, Phys. Rev. B 57, 1505 (1998).

[15] J. P. Perdew, K. Burke, and M. Ernzerhof, Phys. Rev. Lett. 77, 3865 (1996).

[16] G. Kresse and J. Furthmüller, Phys. Rev. B 54, 11169 (1996).

[17] G. Kresse and D. Joubert, Phys. Rev. B 59, 1758 (1999).

[18] P. E. Blöchl, Phys. Rev. B 50, 17953 (1994).

[19] B. Meredig, A. Thompson, H. A. Hansen, C. Wolverton, and A. van de Walle, Phys. Rev. B 82, 195128 (2010).

[20] S. Rossignol, Francois Gérard, D. Mesnard, Ch. Kappenstein, and D. Duprez, J. Mater. Chem. 13, 3017 (2003). 